\title{
SEN Y LOS DERECHOS HUMANOS: LA LIBERTAD COMO OBJETO MATERIAL DE LOS DERECHOS HUMANOS
}

\author{
EDUARDO IBÁÑNZ \\ Universidad Loyola Andalucía
}

\begin{abstract}
RESUMEN: Este trabajo expone el enfoque de la capacidad de Amartya Sen como su mayor aportación al pensamiento sobre los derechos humanos. Además de situar el origen y significación de su aporte, pone en relación el enfoque de la capacidad con el pensamiento de liberación latinoamericano de Ignacio Ellacuría, destacando su importancia en relación con una visión intercultural de los derechos humanos. Expone también otras versiones relevantes del enfoque de la capacidad, específicamente la de Martha Nussbaum. Por último, se sitúan las críticas más relevantes al mismo y la repuesta del autor a las mismas.
\end{abstract}

PALABRAS CLAVE: derechos humanos, enfoque de la capacidad, libertad, liberación, interculturalidad, pobreza, desarrollo humano.

\section{Sen and the Human Rights: Freedom as Material Object of Human Rights}

ABSTRACT: Specifically we understand that the capability approach of Amartya Sen, is his greatest contribution to thinking about human rights. In this paper, in addition to placing its origin and significance for them, we will put in relation the capability approach with some contributions of liberation thought on Latin American as Ellacuría, highlighting its importance in connection with an intercultural vision human rights. We will discuss other relevant versions of the capability approach, specifically that of Martha Nussbaum. Finally, the most relevant reviews are located and the author's response to them.

KEY WORDS: human rights, capability approach, freedom, liberation, intercultural, poverty, human development.

\section{Origen y aproximación al enfoque de la capacidad de Amartya Sen}

El nacimiento del enfoque de la capacidad tenemos que buscarlo en las investigaciones de Amartya Sen sobre diferentes aspectos que confluyen en un mismo objetivo dentro de la teoría de la elección social y en particular en la economía del bienestar. Este objetivo es la superación de la visión utilitarista, o de maximización de algo como la opulencia económica como criterio para la consecución de la adecuada información para las elecciones colectivas. Específicamente en sus trabajos sobre las hambrunas, la pobreza, desigualdad económica y sobre el concepto de desarrollo, que dio lugar al de desarrollo humano, es donde se ha ido gestando el núcleo del enfoque de la capacidad. 
Amartya Sen, en 1979 pronuncia en la Universidad de Stanford la conferencia titulada Equality of what ${ }^{1}{ }^{1}$ En el Ciclo de Conferencias Tanner de la Universidad de Stanford que tiene como pretensión hacer avanzar y ser un reflejo del conocimiento científico relacionado con los valores y las apreciaciones humanas ${ }^{2}$. En este trabajo se empieza a desarrollar su enfoque de la capacidad. La primera y principal tesis de este trabajo es que el ideal moral de la igualdad, que propugnan tanto el enfoque utilitarista, como el enfoque de los bienes primarios de John Rawls 3 , serían insuficientes para legitimarse moralmente por los fallos que encontrarían en la consecución de la igualdad perseguida. Dada la diversidad natural y social entre las personas, se debe ampliar la base de información para la búsqueda de la igualdad. Hay muchos y relevantes aspectos que no se tienen en cuenta en ambos enfoques. En el ejemplo propuesto por Sen de la discapacidad encontramos cómo con los mismos medios se obtiene una menor utilidad. Nos encontramos en una situación donde, por la utilidad marginal, para la métrica utilitarista no está justificada una inversión mayor y expresa la dificultad de estas formulaciones para satisfacer estas ansias de igualdad a través de los mecanismos planteados por ellos. Pero el ejemplo no deja de ser eso, un mero ejemplo, pues la diversidad realmente no es un caso excepcional, sino que se halla en las diferencias entre las personas en muchos ámbitos como la salud, longevidad, condiciones climáticas en las que viven, lugar geográfico, condiciones de trabajo, temperamento, tamaño del cuerpo, etc. Por tanto, el problema de estos enfoques se da no sólo en la atención a la discapacidad, sino en la atención a la diversidad y complejidad propia de las personas ${ }^{4}$. La segunda tesis de este trabajo es una aportación constructiva o creativa en el ámbito de la igualdad, aunque Sen recalca que la principal tesis del artículo es la anterior, o sea la refutación de la igualdad del utilitarismo y de los bienes primarios de Rawls. Esta aportación creativa sería la propuesta de la igualdad de las capacidades básicas, que se concretan en que la persona sea capaz de hacer ciertas cosas básicas como desplazarse, satisfacer las propias necesidades alimentarias, disponer de los medios para vestirse y tener alojamiento, o la capacidad de participar en la vida social de la comunidad ${ }^{5}$. Lo que distingue a su propuesta es que en esta igualdad de capacidades básicas la preocupación no está en los medios, ya sea la utilidad, ya sean los recursos primarios, sino que supone un desplazamiento de la atención sobre los bienes a lo que los bienes suponen para los seres humanos ${ }^{6}$.

1 Sen, A., "¿Igualdad de qué?», Libertad, Igualdad y Derecho. Las conferencias Tanner sobre filosofía moral, Ariel, Barcelona, 1998. Conferencia pronunciada en el Ciclo de Conferencias Tanner sobre Valores Humanos,

2 SEn, A., Nuevo examen de la desigualdad. Editorial Complutense, Madrid, 2001, p. XI.

3 Rawls, J. A., A theory of Justice, Harvard University Press, USA, 1999, pp. 78-81.

4 SEN, A., "¿̇Igualdad de qué?», op. cit., pp. 133-156.

5 Ibíd., p. 152.

6 Ibíd., p. 153. 
Este enfoque ha generado, en el ámbito del desarrollo, una reacción a las formas de medición del desarrollo vigentes hasta su formulación, como el producto interior bruto per cápita o la renta. A través de la visión del desarrollo, vinculado a esta medida, se habían producido condiciones en las que efectivamente se había generado crecimiento económico, pero éste no había ido acompañado de una mejora de las condiciones de vida de las personas ${ }^{7}$. Además, este enfoque cuantitativo había supuesto que la política nacional e internacional dirigida al desarrollo se hubiera centrado casi exclusivamente en generar escenarios macroeconómicos estables y favorables para la inversión extranjera, para los cuales se tuvieron que realizar unos recortes y ajustes presupuestarios sociales que afectaron directamente a las condiciones de vida y a las propias posibilidades de desarrollo de las personas afectadas como se ha podido comprobar en la década de los 80 del siglo xx en América latina con las políticas de los programas de ajuste estructural ${ }^{8}$.

A través del enfoque de la capacidad Sen pretende ampliar la base de información para la toma de decisiones colectivas teniendo en cuenta no sólo la renta, que desde luego es relevante, sino teniendo también en cuenta otros elementos relevantes como la salud, la esperanza de vida, educación, alfabetización, acceso a agua potable, género, desigualdad, participación política, autoestima, etc. Su colaboración en la elaboración del Índice sobre Desarrollo Humano (IDH), dirigido por el economista paquistaní Mabub ul-Haq, publicado por primera vez en el año 1990 por el Programa de Naciones Unidas para el Desarrollo, introdujo este enfoque como clave para la consecución de un nuevo índice de medición del desarrollo que dejaría ser puramente cuantitativo, basado únicamente en la renta, y empezaría a incorporar también elementos para valorar otros elementos relevantes para el desarrollo humano como la educación y la salud (esperanza de vida, alfabetismo e ingreso básico) ${ }^{9}$. El desarrollo humano no es un concepto cerrado y el propio Sen admite que el IDH tiene sus limitaciones, fundamentalmente por su simplicidad, pero era necesario para la generación de un nuevo índice aplicable a todos los Estados. Por otro lado, también se constituyó en el año 2004 la Human Development \& Capability Association, Agency, Well-being and Justice, que es actualmente el motor de desarrollo del enfoque de la capacidad, de la cual Amartya Sen fue su primer presidente, y al que siguió Martha Nussbaum, los dos grandes fundadores teóricos del enfoque de la capacidad.

Amartya Sen ha seguido trabajando en muchas de sus obras revisando y aportando mayor coherencia y respondiendo a los debates suscitados por su enfoque. Para comprender básicamente el enfoque de la capacidad como lo expone Sen hay que explicar al menos los siguientes tres conceptos:

\footnotetext{
SEN, A., El nivel de vida, Editorial Complutense, Madrid, 2001, pp. 50-54.

8 Pérez de Armiño, K., Diccionario de ayuda humanitaria y cooperación al desarrollo, Icaria, Barcelona, 2000, pp. 446-448.

9 PNUD, Desarrollo Humano, Informe, Tercer Mundo Editores, Colombia, 1990, p. 34. http://hdr.undp.org/sites/default/files/hdr_1990_es_completo_nostats.pdf
} 
funcionamientos, capacidad y agencia. A continuación también veremos la importancia del enfoque de la capacidad tanto en el análisis sobre la pobreza, como para una correcta comprensión de la misma.

\subsection{Funcionamientos}

En otro de sus primeros trabajos relevantes para el desarrollo del enfoque de la capacidad Commodities and capabilities $(1987)^{10}$ Sen distingue el concepto de bienes del de funcionamientos como crucial para entender qué es lo que está criticando cuando se opone al enfoque de la igualdad basado en los bienes. De nuevo nos pone un ejemplo en el que ante dos personas que poseen la misma cantidad de alimentos, no podemos considerar que están en igualdad de condiciones, puesto que tenemos que analizar sus funcionamientos, es decir, si las dos personas con la misma cantidad de alimentos logran el mismo grado de nutrición en función de sus características personales, de la misma forma que ante un discapacitado con la misma cantidad de bienes y otra persona sin esa discapacidad, no podríamos decir que se encuentran en las mismas condiciones de bienestar desde la perspectiva de Sen. En este sentido, un funcionamiento es un logro o una consecución de una persona: lo que una persona es capaz de hacer o ser, y se refiere al estado de la persona frente a los bienes que posee que pueden servirle para obtener esos funcionamientos. Es, por tanto, diferente a la posesión de bienes y a la obtención de una determinada utilidad en forma de felicidad derivada de ese logro. Así, no es lo mismo el logro de montar en bicicleta, poseer una bicicleta o la utilidad que se obtiene por el uso de la misma, que es posterior al funcionamiento mismo. Las funcionalidades o funcionamientos están directamente relacionados con las condiciones de vida, pues son diferentes aspectos de las mismas ${ }^{11}$. Es en este sentido en el que Sen incorpora la crítica de Marx al fetichismo de los artículos de consumo que, aunque se realizó en un contexto muy diferente al actual, es una crítica relevante en cuanto al planteamiento de la diferencia entre bienes y logros en el enfoque de la capacidad ${ }^{12}$. En La calidad de vida ${ }^{13}$ desarrolla los conceptos de funcionamientos como la noción más primitiva del enfoque de la capacidad y que se refiere a las partes del estado de una persona, concretamente a las cosas que logra hacer o ser al vivir, algunos son muy elementales (estar nutrido o tener buena salud) y otros pueden ser más complejos como alcanzar la auto dignidad o integrarse socialmente, es por tanto, el resultado real en la vida de las personas al transformar sus oportunidades en lo que realmente hacen o $\operatorname{son}^{14}$.

10 Sen, A., Commodities and capabilities, Oxford University Press, New Delhi, 2008.

11 Ibid., pp. 6-8.

12 Sen, A., El nivel de vida, Editorial Complutense, Madrid, 2001, pp. 26-27.

13 SEN, A., "Capacidad y bienestar», La calidad de vida, Fondo de Cultura Económica, México, 1998.

14 Nussbaum, M. y Sen, A, (Comps.), La calidad de vida, op. cit. 


\subsection{Capacidad}

Hemos definido funcionamiento como un logro. Por otro lado, la capacidad es la posibilidad para alcanzar algo. La capacidad son las posibilidades, las verdaderas oportunidades con respecto a la vida que puede llevar una persona, y en este sentido materializan la noción de libertad. Pese a la íntima relación de las funcionalidades con las condiciones de vida, las posibilidades también tienen un papel directo en el nivel de vida que no es del todo independiente del aspecto de la libertad. Por ejemplo, muestra Sen que, si puedo escoger entre los niveles de vida A, B, C y D y escojo A en un supuesto y, en otro supuesto, entre los mismos niveles, sólo es posible para mi escoger A, en principio mi nivel de vida no ha cambiado, pero sería absurdo decir que, en ambas situaciones, ex ante, se disfruta del mismo nivel de vida ${ }^{15}$. Por tanto, tenemos que el concepto de capacidad de una persona refleja las combinaciones alternativas de los funcionamientos que esta persona puede lograr y entre las cuales debe darse una elección ${ }^{16}$. En Desarrollo y Libertad, cuando Sen expone los fines y los medios del desarrollo, concibe el desarrollo como un proceso de expansión de las libertades reales de las que disfrutan los individuos. De esta manera, las libertades para Sen tienen un sentido instrumental y un sentido final, puesto que el propio proceso de expansión de las libertades se realiza a través del ejercicio de las mismas ${ }^{17}$.

En Capacidad y bienestar, trabajo que aporta Sen al libro editado con Martha Nussbaum La Calidad de Vida, es donde explicita los vínculos y los contrastes aristotélicos, pues el concepto de capacidad como acabamos de definirlo tiene una analogía directa con el concepto de potencialidad de Aristóteles ${ }^{18}$. Sen reconoce que su inspiración inicial la toma de Adam Smith y Karl Marx, y que es Martha Nussbaum quien desarrolla acertadamente la vinculación aristotélica del enfoque de la capacidad. Aunque, por otro lado, Martha Nussbaum pretende profundizar el carácter aristotélico, avanzando hacia una mayor objetivación del enfoque, realizando un listado de capacidades que Sen entiende que aporta, pero que no es necesario para mantener el enfoque de la capacidad por razones que veremos más adelante con mayor detenimiento, pero que básicamente consisten en no dejar el enfoque cerrado y sin posibilidad para que pueda ser interpretado y completado desde diferentes enfoques sustantivos.

\subsection{Agencia}

Por último, la agencia nos está haciendo referencia a la posibilidad de la materialización o ejercicio de esa capacidad. En primer lugar, para abrir el concepto de agencia hacia el contenido real de la libertad, Sen va configurando las

15 Sen, A., Nuevo examen de la desigualdad, op. cit., p. 55.

16 Ibid., p. 55.

17 Sen, A., Desarrollo y Libertad, op. cit., pp. 55-57.

18 Sen, A., "Capacidad y bienestar», Nussbaum, M.; Sen, A., (eds.), La calidad de vida, op. cit., pp. 74-78. 
opciones de manera que las elecciones, el ejercicio de la agencia, van más allá de la mera búsqueda del bienestar, entendido éste como aumento de los bienes o maximización de la utilidad, y en donde podemos encontrar motivaciones que van más allá de la mera búsqueda del interés personal del homo oeconomicus. En cuanto a la agencia, la libertad de ser agente de una persona se refiere a lo que una persona es libre de hacer y conseguir en la búsqueda de cualesquiera metas o valores que considere importantes ${ }^{19}$. Es, por tanto, una libertad amplia que tiene también en cuenta la concepción del bien de la persona. Para entender en su amplitud el concepto de agencia tenemos que destacar que la persona debe ser agente, tanto en la en la propia elección final, como en la valoración de los criterios que determinan a la misma. El agente, para Sen, no hay que entenderlo de la manera en que se entiende habitualmente en los negocios, es decir, como una persona que representa a otras, sino en un sentido más antiguo: como la persona que actúa y provoca cambios y cuyos logros pueden juzgarse en función de sus propios valores y objetivos, independientemente de que los evaluemos o no en función también de algunos criterios externos ${ }^{20}$.

Esta triple diferenciación —-funcionamientos, capacidad, agencia- nos lleva a su vez a distinguir entre los siguientes cuatro conceptos: logro de bienestar y logro de agencia, libertad de logro y libertad de agencia que evalúan diferentes espacios. Estas cuatro categorías nos sirven para distinguir la evaluación del bienestar, de la evaluación del logro de los objetivos, distintos del propio bienestar que una persona se propone en su vida. En los dos primeros estamos expresando logros en cuanto al aspecto de bienestar y de ejercicio de la libertad (agencia), y en los dos últimos estamos hablando de las posibilidades abiertas y, por tanto, de la libertad de poder optar entre diferentes posibilidades para alcanzar el bienestar y nuestra propia agencia que, aún estando íntimamente relacionadas, podemos distinguir claramente entre ellas ${ }^{21}$.

\subsection{Pobreza como privación de capacidades}

Uno de los usos, y causa del propio enfoque de la capacidad, se ha dirigido directamente a la lucha contra la pobreza, y contra la concepción de la pobreza únicamente como falta de ingresos. Una vez vistos los conceptos anteriores que configuran el enfoque de la capacidad, podemos entender mejor cuál sería la situación de pobreza que hay que revertir y, por tanto, las políticas de reducción de la pobreza deben ir no tanto sólo encaminadas al aumento del ingreso, sino a la expansión de las capacidades y de la agencia de las personas.

El propio concepto de pobreza Sen lo ha ido trabajando desde sus primeras obras sobre la igualdad y las hambrunas, a la vez que ha ido desarrollando el enfoque de la capacidad. Así, la identificación de la pobreza con el ingreso bajo, e

19 Sen, A., Bienestar, Justicia y Mercado, Paidós I.C.E./U.A.B., Barcelona, 1998, pp. 85-86.

20 Sen, A., Desarrollo y libertad, op. cit., p. 35.

21 SEN, A., «Capacidad y bienestar», La calidad de vida, op. cit., pp. 60-62. 
incluso la concepción rawlsiana de los bienes primarios ${ }^{22}$, más compleja e incluyente, sigue siendo insuficiente para la obtención de la buena vida y el aumento de la libertad, al menos por la diversidad personal en relación con la edad, el género, discapacidad, aunque también por el lugar físico, las condiciones ambientales, la influencia de las condiciones sociales, de educación, salud pública o las relaciones comunitarias. En su trabajo Poor relatively speaking de 1983, a través de esta nueva forma de concepción de la pobreza confronta la actual concepción de la pobreza como algo relativo, defendiendo una definición y medición de la pobreza que pueda realizarse en términos absolutos ${ }^{23}$. En este trabajo Sen aborda el debate sobre la tensión entre lo absoluto y lo relativo de la pobreza, por ejemplo, si medimos la pobreza a partir de la renta media, efectivamente nos enfrentamos a la paradoja de que siempre habría pobres, puesto que siempre habrá personas por debajo de esa media, pero si consideramos la pobreza en funcionalidades y posibilidades podemos eliminar una buena parte de esa relatividad.

Desde la perspectiva del enfoque de la capacidad es desde donde podemos realizar un abordaje para la ampliación de las capacidades o de la libertad como eje de lucha contra la pobreza. Para esto tenemos que comprender bien el sentido y alcance de la libertad en el pensamiento de Amartya Sen.

\section{Sen y la libertad. El objeto material de los derechos humanos}

La libertad, en un sentido tradicional, se suele entender en un doble sentido, en su formulación más elaborada por Isaiah Berlin en Dos conceptos de libertad $^{24}$. Por una parte, estaría la libertad negativa como aquella que se corresponde con la que se tiene para realizar alguna acción si no se es impedido por nadie para realizarla, esta libertad puede ser reducida por la acción exterior de otras personas. En segundo lugar, estaría la libertad positiva, en la que lo consustancial es que se es dueño de las propias decisiones, de ser uno mismo y en la que se valora la capacidad de elección desde la propia libertad. Berlin usó su famosa distinción para proyectarla sobre la realidad del mundo enfrentado en bloques atribuyendo a cada uno el ejercicio de un tipo de libertad y en donde la libertad positiva es usurpada por un yo superior o colectivo que acaba anulando el aspecto positivo de la libertad. Berlin opta finalmente por un concepto liberal de la libertad negativa que ha sido defendido históricamente por esta tradición filosófica política del liberalismo. El propio Sen reconoce esta mayor relación de la libertad negativa con el aspecto de proceso de la libertad, que veremos más adelante, en la concepción liberal de los derechos ${ }^{25}$. Pero no

22 Rawls, J., El liberalismo político, Crítica, Barcelona, 1996, pp. 215-220.

23 SEn, A., "Poor relatively speaking», Oxford Economic Papers, New Series, Vol. 35, nº. 2 (Jul., 1983), pp. 153-169.

24 Berlin, I., Dos conceptos de Libertad y otros escritos, Alianza Editorial, Madrid, 2001.

25 SEN, A., Bienestar, Justicia y Mercado, op. cit., p. 133. 
deja tampoco Sen de advertirnos del peligro de esta interpretación de la libertad que puede dar lugar a una justificación del propio mercado no basado en sus efectos de generación de bienestar, sino por ser derechos fundamentales que las personas tienen y que ni el Estado ni las personas deben permitir o impedir. En este marco, para Sen es ciertamente posible que existan hambrunas generalizadas sin que se violen los derechos liberales de nadie y sin que deje de operar un mecanismo de mercado libre, de donde se sigue que la aceptación de los derechos liberales de forma independiente de los resultados puede ser inaceptable éticamente ${ }^{26}$.

Para Sen, sin embargo, las libertades, entendidas desde la perspectiva de la capacidad, conforman el objeto material de los derechos humanos, en su doble aspecto de proceso y de oportunidad ${ }^{27}$. Para el autor las libertades reales, como las entiende y propone en su trabajo Desarrollo y Libertad ${ }^{28}$, las que se suceden en la vida real de las personas, son el contenido material de los derechos humanos. Sen recurre a muchos ejemplos en sus obras para explicar su enfoque sobre la libertad en directa relación con la propuesta del enfoque de la capacidad. Partimos de la exposición de los conceptos de funcionamientos, capacidad y agencia que conforman el núcleo del enfoque, especialmente haciendo referencia a que el ámbito de la libertad se expresa concretamente en la libertad de agencia, o en la libertad del agente.

Para entender correctamente el concepto de libertad en Sen tenemos que empezar por decir que llega a la libertad a través de la búsqueda de la igualdad. En ¿Igualdad de qué? ${ }^{29}$, analiza cómo desde diferentes propuestas éticas sociales se propugnan diversas búsquedas de la igualdad. En este trabajo Sen analiza fundamentalmente las propuestas de libertarios, utilitaristas y liberales igualitarios (Rawls y Dworkin) para concluir que todos estos enfoques son insuficientes. El autor termina proponiendo una igualdad sobre las capacidades básicas que acabará llamando libertad(es) y que son la puesta en marcha de su enfoque de la capacidad. Con el enfoque de la capacidad, además, Sen pretende superar el enfoque ético de la economía del bienestar en el que la igualdad se busca a través de los medios y recursos. El nobel indio muestra que esta propuesta es insuficiente ya que lo importante no son los medios, que evidentemente tienen su importancia, sino las oportunidades reales que tienen las personas y, además, que sean ellas mismas las que tomen las decisiones sobre la vida que desean vivir y tienen razones para valorar. No se trata, por tanto, únicamente de los medios que la persona posea, sino de las capacidades que puede generar y de las oportunidades que la persona tiene. Es un enfoque original en tanto que la propia concepción de la libertad se genera a través de la defensa de la igualdad de capacidades básicas.

26 Ibid., p. 134.

27 SEn, A., "Elements of a Theory of Human Rights», Philosophy \& Public Affairs 32, 4, 2004, p. 319.

28 SEN, A., Desarrollo y libertad, op. cit., pp. 276-299.

29 SEN, A., "¿Igualdad de qué?» op. cit. 
Para comprender el significado de la libertad desde el enfoque de la capacidad también tenemos que atender a su doble aspecto: en su faceta de proceso y en su faceta de oportunidad. El aspecto de proceso de la libertad, clave para entender la libertad en todas sus dimensiones, no se hace notar si sólo evaluamos que la finalidad perseguida coincide con el resultado final en la realidad, por ejemplo, cuando un sujeto quiere salir a la calle y sale por su propia voluntad. Se muestra, sin embargo, cuando evaluamos las posibilidades previas al resultado final, aunque se obtenga el mismo que se deseaba obtener. Siguiendo el ejemplo anterior, se puede observar cuando un sujeto quiere salir a la calle y sale porque es obligado a salir de su casa. En este último supuesto, aunque el sujeto logra lo que deseaba, observamos la violación del aspecto de proceso de su libertad. El aspecto de oportunidad sería únicamente violado si le hubieran impedido salir, supuesto en el que se hubiera violado tanto el aspecto de proceso, cuanto el de oportunidad. Ambos aspectos son los que conforman la idea de libertad desde la capacidad. No sólo son importantes los logros sino las oportunidades que tenemos para elegir aquello que tenemos razones para valorar.

Ambos aspectos son fundamentales para comprender bien la libertad como la entiende Sen. Una persona puede saber mis gustos e incluso puede ser capaz de expresar mejor que yo mismo mis puntos de vista y expresarlos, el objetivo final, el aspecto de oportunidad de la libertad en ambos casos puede ser el mismo, pero no podemos obviar el aspecto de proceso que es aquel que valora el proceso de elección y no sólo el resultado, porque existen buenas razones para valorar este proceso y este aspecto de la libertad. La capacidad es la oportunidad de alcanzar combinaciones valiosas de funcionamientos humanos, aquello que una persona es capaz de hacer o ser. El aspecto de oportunidad desde el enfoque de la capacidad permite distinguir aquello que valoramos de los medios para conseguirlo. El enfoque de las capacidades se fija en las oportunidades actuales que se tienen, y no sólo en los medios.

Las aportaciones de este enfoque pretenden superar como hemos dicho, en primer lugar, a las teorías utilitaristas, y en segundo lugar, a la propia teoría de la justicia de John Rawls. La teoría de la justicia de Rawls resulta insuficientemente igualitaria y Sen focaliza su crítica fundamental en el siguiente aspecto: Rawls basa la igualdad en los bienes primarios, pero existe una dificultad en la conversión estos bienes primarios en capacidades, pues como vimos con el ejemplo de la discapacidad, un discapacitado necesita más medios para poder obtener los mismos resultados que otra persona que no lo es. Una teoría de la justicia que mira únicamente a los medios dejará bastantes temas sin resolver.

\section{LIBERTAD Y LIBERACIÓN}

Hemos considerado oportuno realizar una indicación sobre el concepto de libertad y liberación de Ellacuría, porque entendemos que hay una fuerte sintonía entre el propio concepto de liberación, que se refiere a un proceso de 
capacitación de las personas en la sociedad y en la historia, y el enfoque de la capacidad. Ellacuría trabaja de manera muy completa el concepto de libertad, pero efectivamente para Ellacuría la libertad no es algo dado, sino que es algo que se va adquiriendo procesualmente, y que efectivamente se enfrenta además de a las limitaciones físicas propias de la especie humana y a las propias que una determinada persona pueda tener, con las limitaciones que se producen por el contexto social e histórico concreto en el que se puede ir produciendo este proceso de liberación de las personas y de las mayorías populares ${ }^{30}$. En este sentido hay una cierta analogía con el enfoque de la capacidad como proceso de liberación o ampliación de la libertad, o expansión de las capacidades en términos de Sen. Además, efectivamente este proceso de liberación se produce en la historia, es interesante cómo en Filosofía de la realidad histórica, Ellacuría, siguiendo la antropología de Zubiri, a propósito de la realidad formal de la historia, la presenta como proceso creacional de capacidades. Aquí Ellacuría contrapone el concepto del florecimiento humano, entendido como fruto de un concepto de la historia como maduración natural, frente a la apropiación de posibilidades, más acorde con la concepción ellacuriana de la historia.

Con las estructuras psico-orgánicas, el hombre tiene que hacer su vida, tiene que hacer con su personeidad, una personalidad, y esto no puede concebirse en términos de germinación (...). La vida humana no es florecimiento de algo que está por debajo de ella, sino una apropiación de quien estando por encima hace suyas unas posibilidades que antes no le pertenecían ${ }^{31}$.

Para Ellacuría no son equiparables los términos de libertad y liberación. El primero de ellos sería la libertad de los liberales, del liberalismo. Mientras que la liberación es de todo hombre y de todos los hombres y se aproxima a los procesos de liberación histórica. La liberación en este sentido es un proceso con varias dimensiones. Es un proceso personal donde la libertad personal ha de conquistarse del lado negador y del lado creador. A su vez, es un proceso histórico porque supone un proceso de transformación y, o revolución. No se puede hablar de libertad, por tanto, si no se dan las condiciones económico-sociales y políticas que la hagan posible. Y es también un proceso colectivo, tanto por razón de su sujeto activo como de su sujeto pasivo.

Por tanto, desde la perspectiva ellacuriana no hay libertad sin liberación, y ésta es la respuesta cristiana a la libertad colectiva, que posibilita y potencia la libertad personal que es ante todo, liberación de las necesidades básicas y es liberación de los fantasmas y las realidades que atemorizan y aterrorizan al hombre. Supuestas estas dos, está la liberación, tanto la personal, cómo la colectiva, en simultaneidad con ellas, de todo tipo de dependencias ${ }^{32}$.

30 Ellacuría, I., «Ética fundamental», Senent, J. (ed.), La lucha por la justicia, selección de textos de Ignacio Ellacuría (1969-1989), Universidad de Deusto, Bilbao, 2012, pp. 41-53.

31 Ellacuría, I., Filosofía de la realidad histórica, Trotta, Valladolid, 1990, pp. 421-446.

32 Senent, J. (ed.), La lucha por la justicia. Selección de textos de Ignacio Ellacuría (19691989), op. cit., pp. 127-149. 
En este punto entendemos que la propuesta seniana de contenido material de los derechos humanos como las libertades recogen no sólo el concepto de la libertad liberal burguesa de la que nos habla Ellacuría, sino que también se ajusta a la idea de liberación ellacuriana.

\section{Nussbaum, la Versión política JuRídica del ENFoque de las CAPACIDADES Y LOS DERECHOS HUMANOS}

El trabajo conjunto de Sen y Nussbaum ha sido fundamental para el desarrollo del enfoque de las capacidades tan relevante hoy para el desarrollo humano como para una gran cantidad de estudios y enfoques en todas las ciencias sociales. Sen, a partir del trabajo conjunto con Nussbaum en el programa WIDER de Naciones Unidas ${ }^{33}$, profundiza en la raíz aristotélica del enfoque de las capacidades para dotarle de una mayor consistencia y ha sido a partir de entonces que ambos han mantenido vigorosamente en marcha el enfoque a través de sus trabajos e investigaciones, así como por medio de la asociación mundial de dicho enfoque y que han presidido ambos ${ }^{34}$.

Nussbaum muestra el caso de Vasanti, una mujer india separada de casta media maltratada por su marido, para explicar básicamente en qué consiste el enfoque de la capacidad y cómo este enfoque y no el crecimiento económico ayuda a Vasanti a mejorar su vida. Es decir, el aumento de renta de un determinado territorio, incluso de la misma persona afectada por esta situación puede aportar poco a la mejora de la vida real de esta persona. Frente a esta mejora en los recursos, Nussbaum propone que lo importante es ampliar la libertad de actuación de esta persona, no tanto aumentando sus recursos, que puede ser de gran ayuda, pero que puede que la deje en la misma situación de dependencia y violenta opresión. Por tanto, esta aproximación particular a la evaluación de la calidad de vida y a la teorización sobre la justicia social básica se basa en la respuesta a la pregunta ¿qué es capaz de hacer y ser cada persona? La persona es un fin en sí mismo y el enfoque de la capacidad está centrado en la elección o en la libertad, siendo pluralista en cuanto a valores, se ocupa de la injusticia y las desigualdades sociales arraigadas, asignando una tarea urgente al Estado y a las políticas públicas. ¿Qué son las capacidades? Lo que es capaz de ser y hacer una persona, libertades sustanciales en palabra de Sen, la capacidad hace referencia a las combinaciones alternativas de funcionamientos que le resulta factible alcanzar a una persona determinada. Es una especie de libertad, pero no son simples habilidades pues además, incluyen el entorno

33 UNU/WIDER es el primer centro de investigación creado por la Universidad de Naciones Unidas (UNU) en 1985, en Helsinki (Finlandia). La UNU es una organización académica internacional dedicada a fomentar los objetivos de la ONU, particularmente la paz y el progreso. http://www.unesco.org/unuoe/unuesp/centros/wider.htm

34 Human Development and Capability Association (HDCA). http://hd-ca.org/ 
político, social y económico. Para Nussbaum las capacidades están combinadas, compuestas por las capacidades internas y el entorno. Todas las personas deben superar un umbral de capacidades combinadas para ser tratados con igual respeto. Distingue Nussbaum entre las capacidades básicas: las innatas, sean nutridas y desarrolladas posteriormente o no, y el funcionamiento, que define como haceres y seres, la realización activa de una o más capacidades. La libertad tiene valor intrínseco, y no necesariamente su agencia, conlleva su mejor uso o aprovechamiento (ej. ayuno).

Es fundamental, en el planteamiento del enfoque de la capacidad de Nussbaum, la concreción de las diez capacidades centrales para que la vida esté a la altura de la dignidad humana, que debe ser tarea de un gobierno de un orden político aceptable: 1) Vida, como duración normal de la misma, y que no se vea demasiado reducida. 2) Salud física, buena salud, incluyendo la reproductiva, la alimentación y un lugar apropiado para vivir. 3) Integridad física, poder desplazarse libremente, no sufrir ataques, incluidos entre estos los sexuales, incluso en el ámbito doméstico. 4) Sentidos, imaginación y pensamiento, en un modo verdaderamente humano, a través de una educación adecuada, incluyendo en esta capacidad la experimentación de la producción de obras y actos religiosos, literarios, musicales y similares, en condiciones de libertad de expresión. 5) Emociones, poder sentir apego por cosas y personas, amar, sentir duelo, desarrollo emocional. 6) Razón práctica, como formación de una concepción del bien y una planificación de la propia vida. 7) Afiliación, para poder vivir con y para los demás, y disponer de las bases sociales suficientes para sentir respeto por uno mismo. 8) Otras especies, como relación con el mundo natural próxima y respetuosa. 9) Juego, reír, y disfrutar. 10) Control sobre el propio entorno, político, como poder de participación y material, como poder de poseer propiedades y ostentar derechos en igualdad con las demás personas ${ }^{35}$.

Para Nussbaum es necesario virar hacia el enfoque de la capacidad desde el enfoque de derechos, criticando la división de los derechos humanos en generaciones porque se ha mostrado inoperante en la práctica.

Nussbaum desarrolló el nexo con el pensamiento aristotélico del enfoque de la capacidad que Sen ha reconocido posteriormente en su obra ${ }^{36}$. Para Sánchez Garrido, el pensamiento aristotélico de Sen se muestra en la relación entre la ética y la economía, en la no división entre hecho y valor, en el enfoque en cierto sentido dirigido hacia la felicidad (eudemonismo, florecimiento humano como desarrollo pleno de las capacidades, bien-estar frente al bienestar utilitarista), la defensa de una libertad positiva en el sentido ya visto de Berlin, el carácter instrumental de los recursos frente a los fines humanos, su racionalismo

35 Nussbaum, M., Crear Capacidades. Propuesta para el desarrollo humano, Paidós Estado y Sociedad, Barcelona, 2010, pp. 53-55.

36 Nussbaum, M. y Sen, A. (Eds.), El nivel de vida, op. cit., pp. 74-78. 
y universalismo frente al relativismo ético-cultural, y una cierta concepción comunitaria de la sociedad ${ }^{37}$.

Pero también tenemos que exponer las diferencias que se producen entre los autores del mismo enfoque. Nussbaum se ha centrado en el desarrollo de un listado objetivo de capacidades que debe ser incorporado en los consensos político-constitucionales. El pensamiento de Nussbaum es más puramente aristotélico, mientras que el pensamiento de Sen es pretendidamente más abierto y plural, donde una de sus raíces es la aristotélica pero no es la única y siempre con matices. Sen, por otro lado, no comparte este enfoque puesto que en su perspectiva, se trata de llegar a estos resultados, un listado de capacidades, a través de procesos de discusión, debate, participación pública y no tendría sentido dar una lista objetivada, ya elaborada y cerrada de los mismos. En este sentido para Conill, la lucha contra el relativismo y el subjetivismo que se propone Nussbaum empieza, en primer lugar, con la formulación del listado de las capacidades básicas objetivas, y acaba enmarcándose en la propuesta rawlsiana del liberalismo político y del consenso superpuesto. Por otro lado, discurriría, sin embargo, el enfoque de la capacidad de Sen puesto que ofrece la posibilidad de generar una hermenéutica intercultural de mayor recorrido, y entiende que la vía aristotélica no es la única manera consistente de interpretar el enfoque de la capacidad, ni la naturaleza humana. En opinión de Conill la propuesta seniana de la capacidad es una propuesta transcultural que recoge que en todas las culturas hay elementos de búsqueda de ampliación de la libertad frente al cierre de la cultura sobre sí y sobre este ansia de expansión de la libertad ${ }^{38}$.

Para Sen, el problema no está en tener un listado de capacidades importantes, sino en insistir en un predeterminado listado oficial de capacidades, elegido por teóricos, sin procesos de discusión social o debate público. Teniendo esta lista prefijada se niega la fructífera participación pública sobre qué debería ser incluido y por qué39.

Nussbaum, sin embargo, tiene un enfoque más político-constitucional-legal de las capacidades que Sen no comparte. Como hemos visto la divergencia está en el listado objetivo de funcionamientos y capacidades humanas fundamentales.

Sen comparte con Nussbaum que un listado de capacidades generador de unos derechos básicos, puede ser un instrumento extremadamente útil contra la probreza y la privación puede ser muy eficaz, pero para otros propósitos podemos necesitar un listado diferente ${ }^{40}$. Entiende Sen que ante contextos que

37 Sánchez Garrido, P., Raíces Intelectuales de Amartya Sen. Aristóteles, Adam Smith, Karl Marx, Centro de Estudios Políticos y Constitucionales, Madrid, 2008, p. 62.

38 Conill, J., "Hermenéutica crítica intercultural desde el enfoque de las capacidades», Investigaciones fenomenológicas, $\mathrm{n}^{\circ} .7,2010$, pp. 35-44.

39 SEN, A., «Human Rights and Capabilities», Journal of Human Development, Vol. 6, No. 2, July 2005, p. 9.

40 SEN, A., «Human Rights and Capabilities», op. cit., p. 9. 
pueden variar no podemos aceptar una sustantiva disminución de la prevalencia del razonamiento y del debate público ${ }^{41}$. Para Sen, en todo caso, el enfoque de la capacidad permite considerables diferencias en su aplicación.

\section{CRíticas al ENFoque de la CAPACIDAD}

Uno de los elementos por el que podemos reconocer la relevancia de las aportaciones del enfoque de la capacidad reside en la fuerte influencia que ha tenido en diferentes ámbitos como la filosofía política, la economía, la ética, los derechos humanos, etc. En este sentido, el enfoque de la capacidad ha sido muy rico en la generación de literatura científica en torno al mismo, cosechando asimismo una gran cantidad de crítica. Sería una labor que excedería los límites de este trabajo analizar todas las aportaciones que se han ido haciendo al mismo, por ello vamos a sintetizar las principales críticas realizadas sobre el enfoque de la capacidad de Amartya Sen.

Para realizar esta síntesis, la propia crítica la podemos dividir en tres grupos, el primero de ellos está compuesto por aquellos autores que decididamente apoyan el enfoque de la capacidad pero realizan versiones propias, mejoras o matizaciones sobre el mismo, el principal supuesto de este grupo lo ocuparía el caso de Nussbaum que ya ha sido tratado individualizadamente por su importancia, pero en el que también incluimos a David Crocker y a Sabina Alkire. En un segundo bloque de críticas incluimos a aquellas que reconocen una importante aportación e influencia del enfoque de la capacidad, pero también entienden que existen algunas carencias sustantivas en el mismo que habría que intentar solventar hacia el futuro, en este grupo situamos a Bernard Williams que desde diversas concepciones trata de realizar aportaciones sustantivas que entendemos que pueden modificar al marco propio del enfoque de la capacidad, en la propuesta de Amartya Sen específicamente. En este segundo grupo también incluimos a Enrique Dussel que entabla un diálogo con Sen, puesto que ve tras los informes del Programa de Naciones Unidas para el Desarrollo la generación de una base material para una ética crítica que sirva de apoyo a la ética de la liberación, pero a su vez encuentra carencias en el pensamiento de Amartya Sen respecto del estudio de las causas que generan la pobreza. Y en un tercer bloque, estarían aquellas críticas que realizan una refutación total al enfoque de la capacidad, y que en nuestro trabajo están representadas por un lado, por Thomas Pogge, que continúa y profundiza el enfoque rawlsiano, y por otro, por algunos autores de inspiración marxista como Gerald Cohen, Julio Boltnivik y Rolando Astarita, que descartan la virtualidad del enfoque de la capacidad como nueva forma de evaluación, o como mejor criterio en la búsqueda de la justicia.

${ }^{41}$ SEN, A., «Elements of a Theory of Human Rights», op. cit., p. 333. 
En el primer grupo hemos situado, como hemos indicado, a Nussbaum, a Crocker y a Alkire, entre otros. Crocker, es un defensor del enfoque de la capacidad, que ha desarrollado su investigación sobre la ética del desarrollo global y un nuevo concepto de desarrollo, buscando una ética social y del desarrollo que aporte hacia una meta-ética comprensiva de la diversidad cultural, a través de estudios empíricos, estudios sobre la política y práctica del desarrollo ${ }^{42}$. En opinión de Crocker, Sen y Nussbaum fundan una nueva ética para el desarrollo que pretende superar al enfoque del ingreso, al enfoque de los bienes primarios de Rawls y al enfoque de las necesidades básicas ${ }^{43}$, pero con todo sigue siendo un enfoque que sigue estando ligado al enfoque de las necesidades básicas y los medios por un lado, y por otro genera un lenguaje que es ambiguo y que fácilmente es malinterpretado por su propia ambigüedad y complejidad ${ }^{44}$. Alkire trata de reconducir la crítica que ella misma realiza sobre la falta de operatividad del enfoque de la capacidad, tal como Sen lo formula, puesto que la apertura del enfoque que debe ser consensuado para su concreción, con participación y ejercicio democrático por los participantes en cada ocasión, denotando la falta de una teoría sustantiva, como la que reclamará Williams, generando un enfoque multidimensional de medición de la pobreza y del desarrollo humano ${ }^{45}$.

En el segundo bloque de críticas podemos encontrar a Williams, que reconoce una gran aportación de Sen con el enfoque de la capacidad pero que a su vez encuentra dificultades sustantivas para el desarrollo del enfoque de la capacidad, ya que necesita de un despliegue teórico no realizado aún. La cuestión de fondo es que el posterior desarrollo que pide Williams no sería coherente con la propuesta de Amartya Sen. Así, para Williams, en el concepto del nivel de vida que trata de ofrecer Sen a través del enfoque de la capacidad, hace falta una teoría sustantiva sobre la propia naturaleza humana o una teoría social general para poder completar el enfoque, puesto que en opinión de Williams

42 CRocker, D., «Functioning and capability: The foundations of Sen's and Nussbaum's development ethic», Political Theory, 1992, pp. 584-612.

43 Caracterizamos brevemente el enfoque de las necesidades básicas siguiendo la voz del Diccionario de Acción Humanitaria y Cooperación al desarrollo como una corriente de pensamiento sobre el desarrollo surgida en los 70 que centra su análisis en la satisfacción de las necesidades básicas de las personas, en contraposición al tradicional énfasis en el crecimiento económico(...). Éstas se dividían en cuatro grupos: a) Los mínimos necesarios para el consumo familiar y personal: alimento, vivienda, etc. b) El acceso a servicios esenciales: salud, transporte, educación o agua potable. c) Las referidas a un puesto de trabajo debidamente remunerado. d) Necesidades cualitativas referidas a un entorno saludable y humano, participación en la toma de decisiones, libertades individuales, etc. (...) Entre los autores de tales estudios figuraron Paul Streeten, Shahid Javed Burki, Norman Hicks o Frances Stewart. Pérez DE Armiño, K. (Dir.), Diccionario de Acción Humanitaria y Cooperación al desarrollo, op. cit., pp. 387-389.

44 CRocker, D., "Ética internacional del desarrollo: fuentes, acuerdos, controversias y agenda», Diálogo filosófico, 69, 2007, pp. 389-400.

45 Alkire, S., «Dimensions of Human Development». World Development, Vol. 30, n. 2, 2002 páginas 181-205 y AlKire, S., The capability approach to the quality of life. Background report prepared for the Commission on the Measurement of Economic Performance and Social Progress, 2008. 
estaría demasiado abierta, y por tanto sería recomendable formular al menos las capacidades básicas, en la clave de una misma naturaleza y derechos o convenciones sociales y o culturales. Williams distingue tres conjuntos de capacidades: para todo el mundo, las dirigidas a todos mis intereses, y los dirigidos exclusivamente a los intereses económicos. Para Williams, Sen se ocupa de los segundos principalmente, refuta el último y entiende que el primero queda fuera de lugar por ser demasiado comprensivo ${ }^{46}$. En cuanto a la primera crítica podemos entender que es la que ha realizado Nussbaum con su interpretación aristotélica del enfoque y que para Sen no es equivocada, aunque no necesaria para poder dar cabida a otros enfoques sustantivos. En cuanto al segundo supuesto que enumera, aunque pueda parecer excesivo en cuanto a aspiraciones, la agencia entendida en toda su amplitud comprende este aspecto, Sen acoge que el concepto de nivel de vida frente al de bienestar, más comprensivo del concepto de capacidad, puede situarse entre los niveles dos y tres que menciona Williams. Sen acoge las indicaciones de Williams como relevantes, pero como en otras ocasiones responde a la cuestión de sustantivar con su propuesta del enfoque de la capacidad: no creo que haya que avergonzarse porque algunas de estas cuestiones evaluativas puedan resultar difíciles, controvertidas y en algunos casos, quizá incluso irresolubles (llevando a ordenaciones parciales de niveles de vida $)^{47}$.

En este segundo bloque podemos situar el interesante diálogo de Dussel con Sen sobre la posibilidad de una ética crítica formulada a través del enfoque de la capacidad. Para Dussel, Sen puede ayudar a superar una dificultad que ha tenido la ética de la liberación en cuanto a dotarla de un estatuto científico económico, ayudando a aclarar este tema central de toda ética crítica, en el sentido de advertir una negatividad material como diría Max Horkheimer ${ }^{48}$, pues el PNUD además de medir el desarrollo humano también mide los efectos negativos no intencionales de la economía a través del índice de pobreza humana (IPH), donde de la pobreza no significa sólo falta de bienes básicos, sino ante todo falta de capacidad para la realización efectiva del propio modo de vida concreto, cultural. Sin embargo, Dussel critica que Sen no indique claramente las causas de la pobreza y no habla de relaciones de dominación en las relaciones de producción como origen estructural de la pobreza, que indicaría que Sen no tiene una clara conciencia de estar generando una ética crítica a través de sus avances científicos.

En su libro Hacia una filosofía política crítica ${ }^{49}$, Dussel, uno de los principales autores y fundadores de la filosofía de la liberación, entabla un diálogo con Amartya Sen y su enfoque de la capacidad. Para Dussel en los Informes del PNUD se encuentra lo que él denomina un cripto-principio ético material más

46 SEN, A., El nivel de vida, op. cit., pp. 142-154.

47 Ibid., p. 162.

$48 \quad$ Ibid., p. 138.

49 Dussel, E., Hacia una filosofía política crítica, Desclée de Brouwer, Palimpsesto, Bilbao, 2001, pp. 127-144. 
profundo y amplio que para Dussel va más allá que el propuesto por Amartya Sen de las capacidades para el acceso a la realización del modo de vida que las personas valoran. De lo que se trata, según Dussel, es del criterio universal último que estaría inherente, aunque no explicitado en todos los Informes, de una ética material que incluye y supera los principios del utilitarismo, del bienestarismo e incluso de las capacidades. La vida humana es la que permite la satisfacción o la felicidad del cumplimiento de lo deseado, del propio interés y de las necesidades (el alimento, la casa...). Por tanto, para Dussel a Sen le falta la articulación de un principio ético material universal de mayor profundidad y amplitud tal como Dussel lo encuentra bajo los Informes del PNUD ${ }^{50}$ : El ser humano debe producir, reproducir y desarrollar la vida humana en comunidad en todos sus aspectos o determinaciones, como capacidades de realización de esa vida humana, siempre concretada humanamente en un modo de vida o cultura, que es el horizonte histórico desde donde debe medirse su realización (tanto individual como comunitaria, y en último término de toda la humanidad) ${ }^{51}$.

En nuestra opinión Dussel empieza un diálogo fructífero con Sen en el que podemos reconocer algunos puntos comunes de partida: procedencia de un país con alto índice de pobreza y con un pasado colonial, que le hace tener una visión más compleja de la economía. Además Sen se sitúa en una perspectiva crítica respecto a los presupuestos de la teoría económica tradicional de la teoría del equilibrio racional y de la teoría de la elección racional, ampliando el contenido y el horizonte de la economía dando cabida a otros factores más allá del puro egoísmo.

En el tercer grupo de críticas, incluimos en primer lugar a Pogge ${ }^{52}$, rawlsiano, universalista, institucionalista y defensor del consenso superpuesto como la versión internacional del velo de la ignorancia entre diferentes pueblos y culturas. En su trabajo Can the Capability Approach Be Justified?, reconoce el amplio eco e influencia que ha generado el enfoque en la economía normativa y en filosofía política actual, sin embargo, entiende que el enfoque de los recursos, específicamente el de los principios de justicia de John Rawls es superior como criterio de búsqueda de la justicia social, en la conclusión de su trabajo sobre el enfoque de la capacidad es contundente: Can the capability approach be justified? Can it conceivably deliver at least one candidate public criterion of social justice that would be as clear and as workable and as plausible as the leading resourcist criterion, Rawls's two principles? Would such a capability criterion do better in addressing and highlighting the horrific injustices of the world in which we live? The evidence to-date suggests that the answers to these questions are no. That the capability approach has nonetheless done much to advance the

$50 \quad$ Ibid., pp. 133-134.

51 Ibíd., p. 134.

52 Pogge, T., «Can the Capability Approach Be Justified?», Philosophical Topics 30 (2), 2010, pp. 167-228. 
discussion of social justice is a great tribute to its foremost champions: Martha Nussbaum and Amartya Sen ${ }^{53}$.

Para Keleher, Pogge realmente realiza la crítica sobre una mala comprensión del enfoque de la capacidad, puesto que Pogge está concediendo un papel instrumental similar al de los recursos al enfoque de la capacidad ${ }^{54}$. Como respuesta de la propuesta de Sen desde esta perspectiva nos remitimos a la crítica al enfoque rawlsiano, por su abstracción y su limitación a los recursos como medio de trabajar por la igualdad, ya que no es un enfoque suficiente para poder acoger y superar las diferencias que suponen que las distintas situaciones de las personas en la conversión de esos bienes en capacidades o libertad real.

En el tercer grupo de críticas al enfoque de la capacidad hemos indicado que tenemos varios autores de inspiración marxista y que aunque ponen el acento en diferentes cuestiones confluyen en una mayor rotundidad crítica respecto al enfoque de Sen. En cuanto a estos autores, por su mayor relevancia, hemos incluido en primer lugar a Cohen, que reconoce los aportes del enfoque, pero también encuentra varias dificultades en el mismo. En primer lugar, la ambigüedad terminológica. Cohen destaca el descubrimiento de Sen de una métrica a medio camino entre un enfoque sobre los bienes y sobre la utilidad, denominado capacidad por Sen, pero entiende Cohen que no ha desplegado bien su teoría y que es ambigua, para ello propone un concepto más concreto y limitado para recoger la aportación de esta nueva métrica —-midfare ${ }^{55}$-, puesto que entiende que Sen ha encontrado un espacio entre la medición de los propios bienes y la medición de la utilidad producida por los mismos. Además, entiende que Sen se confunde ampliando excesivamente el concepto de libertad, y se enfoca finalmente más hacia la igualdad de las capacidades básicas en un enfoque que va tendiendo hacia los medios finalmente ${ }^{56}$. Para Sen, efectivamente existe ese espacio en el enfoque de la capacidad pero en su opinión, Cohen está confundiendo este midfare o vía media con lo que Sen denomina funcionamientos de una persona y no refiriéndose realmente a la capacidad.

Boltnivik realiza un profundo estudio sobre la evolución del enfoque de la capacidad en Amartya Sen desde su primera versión en Equality of what?, hasta su versión más acabada en Inequality reexamined, y a los desarrollos de alguno de sus colaboradores como Alkire y Foster. También se enfrenta a las principales críticas al enfoque de la capacidad de Williams y Cohen, como parte de un trabajo más amplio sobre la medición de la pobreza. Finalmente, Boltnivik llega a la conclusión de que el enfoque de la capacidad de Sen realmente está centrado en los bienes y es un enfoque mecanicista, puesto que las capacidades dependerían finalmente, de manera implícita, de la consecución de los bienes. Por otro lado, advierte que el enfoque de Nussbaum podría ampliar ese marco,

53 Pogge, T., «Can the Capability Approach Be Justified?», op. cit., p. 71.

54 Keleher, L., Empowerment and International Development. ProQuest, 2007, pp. 70-89.

55 Cohen, G., «Equality of what? On Welfare, Goods, and Capabilities», en Nussbaum, M. y SEN, A., The quality of life, op. cit., p. 18.

56 Ibíd., pp. 9-29. 
pues saca al enfoque de esta unión exclusiva hacia los bienes hacia otras concepciones más sustantivas. Desde esta perspectiva, sitúa al enfoque de la capacidad de Sen como un enfoque mecanicista en la misma línea que la teoría del consumidor, donde prima el individualismo y la motivación de maximización de la utilidad total ${ }^{57}$.

Dean realiza una doble crítica. En primer lugar, por el supuesto liberalismo individualista de la propuesta del enfoque de la capacidad refutándolo, puesto que en el enfoque de la capacidad no se tiene en cuenta la determinante interdependencia de las relaciones sociales. Y en segundo lugar, ya que no podemos excluir las consecuencias del sistema de relaciones de producción capitalista, ni de la explotación del trabajo humano. Parece a su juicio más adecuado, frente al enfoque de la capacidad, un enfoque de lucha por los derechos que enfatice la génesis histórica y de lucha de los mismos ${ }^{58}$. Astarita, en un sentido análogo, concluye que la propuesta de la capacidad y de la democracia reflexiva de Sen no será posible mientras exista la propiedad privada de los medios de producción y critica que no haga Sen un análisis de las causas del origen de la pobreza o sobre cómo se puede conseguir el fin de la pobreza ${ }^{59}$.

Amartya Sen, en su última y más completa publicación sobre su pensamiento, La idea de la justicia, dedica específicamente la tercera parte al enfoque de la capacidad, aunque efectivamente como parte de una teoría de la justicia más amplia, pero en la que va a jugar un papel fundamental, y en la que el propio nombre de esta tercera parte Los materiales de la justicia ${ }^{60}$, nos da una clave interpretativa sobre cuál es el papel del enfoque de la capacidad en su idea de la justicia. Concreta y explícitamente, para que no se malinterprete el enfoque de la capacidad, Sen va a comentar tres características del mismo, una de ellas especialmente relevante en cuanto a una respuesta a la crítica expuesta por este tercer grupo de autores y que Sen denomina el lugar de los individuos y las comunidades y sus interrelaciones en la concepción de las capacidades. Sen trata de responder a las críticas que entienden y sitúan al enfoque de la capacidad bajo la influencia maligna del individualismo metodológico ${ }^{61}$. En primer lugar, entiende que aquellas críticas que identifican el enfoque de la capacidad con el individualismo metodológico cometen un error significativo, ya que el enfoque no asume la separación entre el individuo y la sociedad, y además, porque se preocupa tanto de las influencias sociales, en tanto parte de la comunidad,

57 BoltniviK, J., «Evaluación crítica del enfoque de "capabilities" de Amartya Sen (Primera parte)», Mundo siglo XXI, número 12, primavera 2008, pp. 43-55 y «Evaluación crítica del enfoque de "capabilities" de Amartya Sen (Segunda parte)», Mundo siglo XXI, número 13, verano 2008, pp. 20-41.

58 Dean, H., "Critiquing capabilities: the distractions of a beguiling concept», Critical Social Policy 29-2, 2009, pp. 261-278.

59 Astarita, R., Sen y Marx, http://www.iade.org.ar/uploads/c87bbfe5-c9d8-4172.pdf. Última consulta 22 de mayo de 2014.

60 SEN, A., La idea de la justicia, op. cit., p. 253.

${ }^{61}$ Ibíd., p. 274. 
como de la relevancia del razonamiento público en la propia evaluación individual, rechazando de modo inequivoco toda visión separada de los individuos respecto de sociedad que los rodea ${ }^{62}$. En este sentido Sen resalta que actualmente la tendencia es a identificar de manera simple a los individuos, resaltando únicamente una de sus características, cuando lo característico de los seres humanos es la convivencia de diversas identidades y filiaciones (de género, clase, lenguaje, profesión, nacionalidad, comunidad, raza, religión, etc. ${ }^{63}$.

\section{Conclusiones}

El origen del enfoque de la capacidad se sitúa, por un lado, en la investigación sobre filosofía moral de Amartya Sen sobre la igualdad y, por otro, en la búsqueda de nuevas concepciones de la privación y la pobreza.

Para el autor, la libertad entendida como capacidad tiene una relación directa con los derechos humanos, puesto que desde el enfoque de la capacidad, la libertad, sería el objeto material de los derechos humanos. A nuestro juicio su propuesta ética de los derechos humanos, es su mayor aportación al pensamiento sobre los derechos humanos. El enfoque de la capacidad parte de la diferenciación entre la propia capacidad de la persona, de los medios y de las oportunidades reales que finalmente puede disfrutar la misma, que no son final, ni necesariamente coincidentes con las opciones que una determinada persona realiza históricamente. En el enfoque de la capacidad, la agencia de la persona es un elemento fundamental, su propio ejercicio y desarrollo debe constituir el propio proceso de desarrollo del mismo.

El concepto clásico de libertad, criticado específicamente por el pensamiento de liberación latinoamericano, propone una versión de la libertad que no sea la liberal burguesa para un segmento de la población sino un proceso histórico de consecución de una libertad real para todas las personas y en todas sus dimensiones. Entendemos que la aproximación a la libertad de Sen está más cercana al concepto de liberación en cuanto proceso histórico complejo de apropiación de capacidades y oportunidades que se dan en un contexto socio económico y geográfico concreto, que al concepto abstracto de la libertad en referencia a la libertad del liberalismo.

Nussbaum muestra un desarrollo del enfoque de la capacidad como superación de los derechos humanos con un listado de capacidades básicas de profundo carácter aristotélico. Sen, sin embargo, busca una conexión entre el enfoque de la capacidad y los derechos humanos incorporando a los mismos su propuesta de la capacidad que consolida una visión compleja e histórica de los mismos y que permite un desarrollo intercultural de mayor potencialidad y calado.

62 Ibid., p. 275.

63 Ibíd., p. 277. 
La mayor parte de las críticas al enfoque de la capacidad son aportaciones complementarias al mismo, salvo aquellas que tratan de enmarcar al enfoque de la capacidad dentro del paradigma del individualismo metodológico y del que expresamente se distancia observando el necesario carácter relacional del enfoque entre los individuos y la sociedad que los acoge.

\section{BibliografíA}

Alkire, S., «Dimensions of Human Development», World Development Vol. 30, No. 2, 2002 páginas 181-205.

ALKIRE, S., The capability approach to the quality of life. Background report prepared for the Commission on the Measurement of Economic Performance and Social Progress, 2008.

AstaRita, R., Sen y Marx, http://www.iade.org.ar/uploads/c87bbfe5-c9d8-4172.pdf. Última consulta 22 de mayo de 2014.

BoltniviK, J., "Evaluación crítica del enfoque de "capabilities” de Amartya Sen (Primera parte)», Mundo siglo XXI, número 12, primavera 2008, páginas 43-55.

BoltnIVIK, J., «Evaluación crítica del enfoque de "capabilities” de Amartya Sen (Segunda parte)», Mundo siglo XXI, número 13, verano 2008, páginas 20-41.

CoHEn, G., «Equality of what? On Welfare, Goods, and Capabilities», en Nussbaum, M. y Sen, A. (comps.) The quality of life, Oxford University Press, 1993

Conill SANCHO, J., «Hermenéutica crítica intercultural desde el enfoque de las capacidades», Investigaciones Fenomenológicas, número 7, 2010.

Corominas, J., Ética Primera. Palimsesto. Desclée de Brouwer. Bilbao. 2000.

Crocker, D. A., «Functioning and capability: The foundations of Sen's and Nussbaum's development ethic». Political Theory, 1992

CROCKER, D. A., «Ética internacional del desarrollo: fuentes, acuerdos, controversias y agenda». Diálogo filosófico, 69, 2007, páginas 389-400.

Dean, H., "Critiquing capabilities: the distractions of a beguiling concept». Critical Social Policy 29-2, 2009, páginas 261-278.

Dussel, E., Hacia una filosofía política crítica. Desclée de Brouwer, Palimsesto. Colección Derechos Humanos y Desarrollo. Bilbao. 2001

Ellacuría, I., Filosofía de la realidad histórica. Trotta. Valladolid. 1991.

Keleher, L. W., Empowerment and Development. ProQuest, 2007.

Nussbaum, M. C., Sen, A. (eds.), La calidad de vida. Fondo de Cultura Económica. México. 1998.

Nussbaum, M. C., Crear capacidades. Propuesta para el desarrollo humano. Paidós. Madrid. 2012.

Pérez de Armiño, K., Diccionario de ayuda humanitaria y cooperación al desarrollo. Icaria. Barcelona. 2000

Pogge, T. W., «Can the Capability Approach Be Justified?», Philosophical Topics 30 (2), 2010, páginas 167-228.

Rawls, J., A theory of Justice, Harvard University Press, USA, 1999

SÁnchez Garrido, P., Raíces Intelectuales de Amartya Sen. Aristóteles, Adam Smith, Karl Marx. Centro de Estudios Políticos y Constitucionales. Madrid. 2008.

SEN, A., "Poor relatively speaking», Oxford Economic Papers, New Series, Vol. 35, No. 2 (Jul., 1983), páginas 153-169. 
— «Capability and Well-Being», The Quality of life. Clarendon Press, 1993, páginas 35-36.

- "¿Igualdad de qué?». Libertad, igualdad y derecho: las conferencias Tanner sobre filosofía moral. Planeta Agostini. 1994.

- Nuevo examen de la desigualdad. Alianza Economía. Alianza Editorial. Madrid. 1995

- Bienestar, Justicia y Mercado. Paidós. ICE/UAB. Barcelona. 1998.

- Desarrollo y libertad. Planeta. Barcelona. 2000.

- El nivel de vida. Editorial Complutense. Madrid 2001.

- «Elements of a theory of human rights». Philosophy\&Public Affairs. 32, n 4, 2004, pp. 315-356.

- «Human Rights and capabilities». Journal of Human development. Volume 6, n 2, July 2005.

- Commodities and capabilities. Oxford University Press. New Delhi. 2008.

- La idea de la Justicia, Taurus, Barcelona. 2010.

SEnEnt, J. A. (ed.), La lucha por la justicia, selección de textos de Ignacio Ellacuría (19691989). Universidad de Deusto. Bilbao. 2012.

[Artículo aprobado para publicación en noviembre de 2014] 